\title{
Multi-ring PSK Constellation Design for Spatial Modulation Transmission
}

\author{
P. Zhang, C.D. Wang \\ School of Computer Engineering, Weifang University \\ Weifang, China
}

\author{
G.Y. Xu \\ ShanDong labor vocational and technical college \\ Jinan, China
}

\begin{abstract}
Spatial Modulation (SM) is a recently emerged MultipleInput Multiple-Output (MIMO) technique, which has been proved to be low-complexity and more energy-efficient compared with classical MIMO techniques. As an entirely novel hybrid modulation scheme, the performance of SM is highly dependent on the specific type of digital modulation schemes, and the digital modulation constellation optimizing is still a problem. In this paper, a novel digital modulation constellation design method suitable for SM transmission is proposed, which has multi-ring structure with each ring's constellation similar with PSK constellation. Both analysis and simulation results show that the novel designed multi-ring constellation could provide superior performance compared with traditional PSK and QAM over independent Rayleigh fading channels.
\end{abstract}

Keywords-spatial modulation; multi-ring PSK; MIMO; Rayleigh channels

\section{INTRODUCTION}

Spatial modulation (SM) is a recently proposed multiantenna transmission technique [1]. As an entirely novel hybrid modulation scheme, SM exploits both space and signal domains to modulate bit information. And hereby, for the same spectral efficiency, the cardinality of digital modulation will becomes less, and the system performance could be improved because of enlarged minimum Euclidean distance between constellation points. Furthermore, in SM only a single or a few transmit antennas are activated at each time instant, thus the transceiver complexity could be reduced significantly. Advantages of SM over traditional MIMO schemes have been addressed in the literatures [2], [3]. Therefore, SM aided MIMO (SM-MIMO) has been considered as a candidate technology for next generation wireless mobile communication systems, in particular, when large-scale MIMO is employed [4]

In a traditional single-input single-output (SISO) system, PSK (Phase Shift Keying) or QAM (Quadrature Amplitude Modulation) constellations based on maximum-minimum distance (MMD) is preferred. However, as an entirely novel hybrid modulation scheme, the performance of SM is highly dependent on the specific type of digital modulation schemes, and the advantages of PSK or QAM may be eroded in SM transmission [4-8]. In [5], the bit error probability of SM is derived and its results have shown that PSK may be better than QAM in SM. Furthermore, new researches in [7] found that 4PSK will become the best choice when the number of transmit antennas become unlimited. However, as far as we know for the given cardinality of digital modulation, there are still no clear answers on the preferred constellations. In recently, more researches focus on the design of new APM (Amplitude Phase
Modulation) constellation suitable for SM, just because APM reflects the more general case in two-dimensional modulation schemes. Without loss of generality, APM constellations with multi-ring structure are usually selected in order to facilitate the actual design process. For instance, in [4] and [6], the star-PSK constellation is studied for SM, and its research is based on the former research results in [9], where the authors have shown that the star-PSK constellation could provide better performance than traditional QAM constellation. However, in [9] the radius of the rings in star-PSK is fixed, and aiming at this problem, the literature [4] and [6] extended the research and proposed one method to design star-PSK constellation based on optimizing the rings' radius. Unfortunately, the discussed star-PSK constellation in [4] and [6] still reflected some special cases. And the design method is really difficult to be extended to the constellations with multiple rings and with each ring containing varying number of points.

Therefore, the digital modulation optimizing is still a problem for SM, especially for high spectrum efficiency. In this paper, we propose one new APM constellation design method suitable for SM. Different from the previous researches, the new designed APM constellation will have multiple rings and with each ring containing different number of points, which could reflect more general cases. Furthermore, in order to facilitate the design process, at each ring, the signal points construct one PSK constellation. Therefore, this new class of APM constellations are called as Multi-ring PSK constellation.

\section{MULTI-RING PSK CONSTELLATION DESIGN}

\section{A. Performance of SM with APM Constellations}

In [7] the approximate union bound of average symbol error rate (SER) for SM with APM has been derived at high SNR regions, which is expressed as

$$
P_{N r}(M) \approx\left(\begin{array}{c}
2 N r-1 \\
N r
\end{array}\right)(8 \gamma)^{-N r} G_{N r}(M)
$$

with

$$
G_{N r}(M)=\frac{2^{N r}}{M}\left\{(N t-1) \sum_{\substack{m=1 \\ m^{\prime}=1}}^{M}\left(\left|s_{m}\right|^{2}+\left|s_{m^{\prime}}\right|^{2}\right)^{-N r}+\sum_{\substack{m=1, m^{\prime}=1 \\ m \neq m^{\prime}}}^{M}\left(\left|s_{m}-s_{m^{\prime}}\right|^{2}\right)^{-N r}\right\} \text {. }
$$

Wherein, $P_{N r}(M)$ denotes the approximate average SER. $M$ is the cardinality of APM constellation. $\mathrm{Nt}$ and $\mathrm{Nr}$ represent the number of transmit and receive antennas, respectively. $\gamma$ is the received average signal-to-noise (SNR) ratio. $s_{m}$ and $s_{m^{\prime}}$ denotes APM signal points. 
Note that (1) and (2) are only valid for independent Rayleigh fading channels. Obviously, (1) and (2) are the evaluation criteria for the design of APM constellation suitable for SM. And better APM should have lower $P_{N r}(M)$.

According to (1) and (2), for given $M, N t, N r$ and $\gamma$, $P_{N r}(M)$ is only determined by the location of APM signal points. And hereby, the APM constellation design is to locate APM signal points in two-dimensional space. However, it is impossible to design one good APM by just referring to (1) and (2), so more reasonable restrictive conditions should be given.

\section{B. Design Criteria for Novel Multi-ring PSK Constellation}

In this paper, the goal is trying to find one good APM constellation for given $\mathrm{M}, \mathrm{Nt}$ and $\mathrm{Nr}$. From the point of view of maximizing the minimum Euclidean distance between modulation symbols, as well as to facilitate the design process, we propose the following design criteria.

Criteria 1: Without loss of generality, APM constellation is supposed to have multi-ring structure. Moreover, we assume that the signal points located in the same ring construct one PSK constellation. In other words, the contained signal points in each ring are located at equal space intervals. In the following we call this multi-ring APM constellation as multiring PSK constellation.

Criteria 2: Considering that the ring with larger radius will have longer circumference, and hereby more signal points may be contained for the given minimum Euclidean distance. So, we suppose that the point number in the ring with larger radius is always no less than that in the rings with smaller radius. Of course, the last ring should be excluded.

Criteria 3: From [7], we have known that with $M$ no more than 4 the optimum constellation is really MPSK, furthermore, the performance of SM with 4PSK is really superior to the performance of SM with BPSK. So for each ring, we suppose that the point number will be no less than 3 . And then the ring number of multi-ring PSK constellation (denoted by $n$ ) satisfy

$$
1 \leq n \leq \operatorname{ceil}(M / 3)
$$

Here, the function ceil $(x)$ rounds $x$ up to the nearest integer, if necessary.

Criteria 4: Suppose that two adjacent rings contain $N_{1}$ and $N_{2}$ signal points, respectively. In order to maximize the minimum Euclidean distance between symbols as large as possible, the minimum angle between any two symbols with them located in two adjacent rings is given by

$$
\theta=\pi / \operatorname{LCM}\left(N_{1}, N_{2}\right) .
$$

In which the function $\operatorname{LCM}\left(N_{1}, N_{2}\right)$ is to compute the LCM (least common multiple) of two numbers $N_{1}$ and $N_{2}$.

Criteria 5: The minimum Euclidean distance of the whole multi-ring PSK is determined by the minimum Euclidean distance between symbols in the first ring.
With the above criteria, the design process of novel multiring PSK constellation is expressed as figure 1.

In Step 1, the transmitter should firstly get the MIMO system parameters: $M, N t$ and $N r$. Especially for the number of receive antennas $N r$, the transmitter should get it from the feedback channel in the initial stage of user access.

In Step 2, according to Criteria 3, the maximum ring number of novel multi-ring PSK is calculated as $n=\operatorname{ceil}(M / 3)$. And then the novel multi-ring PSK is designed by a searching process based on criteria 1, 2, 3, 4 and 5 .

In Step 3, the best multi-ring PSK constellation is achieved by selecting the constellation with the smallest $G_{N r}(M)$ value as shown in (2).

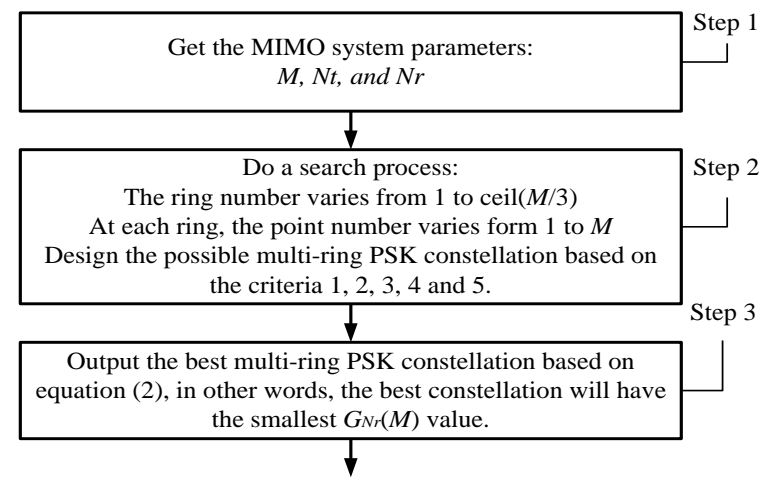

FIGURE I.THE SEARCHING PROCESS OF NEW MULTI-RING PSK CONSTELLATION.

It should be noted that the core of the searching process is Step 2, which is described in details as follows.

In Step 2, the ring number varies from 1 to $n$ (ceil $(M / 3))$, and the point number in each ring varies from 1 to $M$. Obviously, the searching process is similar with one $(M+1)$ hexadecimal number, which has $n$ bits with each bits representing one ring of multi-ring PSK, and the value of each bit denotes the point number contained in the corresponding ring. For example, suppose $M=16$, corresponding to the searching process, we design one 17 hexadecimal number with $6(n=\operatorname{ceil}(M / 3))$ bits. And then for each 6 bits 17 hexadecimal number, it really represents one point number distribution for a multi-ring PSK constellation. For instance, the 6 bits 17 hexadecimal number, 123456, accounts for that the multi-ring PSK constellation have 6 rings, and from the first to the sixth ring, the point number is $1,2,3,4,5$ and 6 , respectively.

Next, suppose that $(M+1)$ hexadecimal number is represented by $i_{1} i_{2} \ldots i_{j} \ldots i_{n}$ with $i_{j}$ denoting the $j$ th bit. And suppose that the ring with the smallest radius is the first ring, which is corresponding to $i_{1}$; the ring with the second smallest radius is the second ring, which is corresponding to $i_{2}$, and so on, $i_{j}$ is corresponding to the $j$ th ring of multi-ring PSK constellation. Furthermore, the value of $i_{j}$ denotes the constellation point number in the $j$ th ring of multi-ring PSK 
constellation. It should be noted that for $i_{1} i_{2} \ldots i_{j} \ldots i_{n}$ not every number is legal. The legitimate number should satisfy the following rules.

(1) $\sum_{j=1}^{n} i_{j}=M$. i.e., the total points' number should be equal to $M$.

(2) $3 \leq i_{1} \leq i_{2} \leq i_{3} \ldots \leq i_{n-2} \leq i_{n-1}$ based on Criteria 2 and 3 .

Therefore, the searching process means the value of $i_{1} i_{2} \ldots i_{j} \ldots i_{n}$ varies from $\underbrace{0000 \ldots 0}_{n \text { bits }}$ to $\underbrace{3333 \ldots 3}_{n-1} X$ bits , and the last bit $X=M-3 \times(\operatorname{ceil}(M / 3)-1)$.

At this point, the searching process could be divided into two sub-steps. The first sub-step is to find one legitimate $(M+1)$ hexadecimal number with $n$ bits. The second sub-step is to locate the constellation points in two-dimensional space based on the given legitimate number $i_{1} i_{2} \ldots i_{j} \ldots i_{n}$. In the second sub-step, the constellation is designed by the following process.

Firstly, suppose the radius of the first ring, denoted as $r_{1}$, is equal to 1 , and then locate $i_{1}$ point in the first ring with equal space intervals, i.e., the points' angles in the first ring are given by

$$
\theta_{1, p}=\frac{2 \pi}{i_{1}} p+\theta_{1,0}, \quad p=0,1, \ldots, i_{1}-1
$$

Here, for searching simplification, the first point's angle in the first ring is set to be zero, i.e., $\theta_{1,0}=0$. And then calculate the minimum Euclidean distance between symbols in the first ring, which is denoted by $d_{\min }$.

Secondly, suppose that the $l$ th $(l=1,2, \ldots, n-1)$ ring's points have been located, and its first point's angle is $\theta_{l, 0}$. According to Criteria 4 , the first point's angle in the $(l+1)$ th ring could be calculated as

$$
\theta_{l+1,0}=\left(\pi / \operatorname{LCM}\left(i_{l}, i_{l+1}\right)\right)+\theta_{l, 0}
$$

Moreover, on the condition of equal space interval the other points' angles in the $(l+1)$ th ring are given by

$$
\theta_{l+1, p}=\frac{2 \pi}{i_{l+1}} p+\theta_{l+1,0}, \quad p=0,1, \ldots, i_{l+1}-1
$$

Next, suppose that the radius of the $l$ th $(l=1,2, \ldots, n-1)$ ring is $r_{l}$, based on $d_{\min }$ we could get the radius of the $(l+1)$ th ring, dented as $r_{l+1}$, by solving the following equations.

$$
\left\{\begin{array}{l}
d_{1}^{2}=r_{l+1}^{2}+r_{l}^{2}-2 r_{l} r_{l+1} \cos \left(\frac{\pi}{\operatorname{LCM}\left(i_{l}, i_{l+1}\right)}\right) ; \quad d_{2}^{2}=2 r_{l+1}^{2}\left(1-\cos \left(\frac{2 \pi}{i_{l+1}}\right)\right) \\
\min \left(d_{1}^{2}, d_{2}^{2}\right)=d_{\text {min }}^{2} ; \quad r_{l+1}^{2}>r_{l}^{2}
\end{array}\right.
$$

Herein, the function $\min (x, y)$ returns the smaller value between $x$ and $y$.

Therefore, once the first ring's parameters have been determined, according to the design criteria proposed in this paper, the other ring's parameters could be obtained easily.

At last, the actual signal amplitude could be achieved by the signal energy normalization based on the whole constellation.

At this point, for one given legitimate number $i_{1} i_{2} \ldots i_{j} \ldots i_{n}$, one multi-ring PSK constellation could be designed. And hence, by varying the value of $i_{1} i_{2} \ldots i_{j} \ldots i_{n}$ from $\underbrace{0000 \ldots 0}_{n \text { bits }}$ to $\underbrace{3333 \ldots 3}_{n-1} X$ bits we hope to get one possible multi-ring PSK constellation superior to traditional PSK or QAM.

\section{Design Examples for Novel Multi-ring PSK Constellation}

In this section, several designed multi-ring PSK constellations are provided with different MIMO system parameters. They are shown in table 1 .

TABLE I. NEW DESIGNED MULTI-RING PSK CONSTELLATIONS.

\begin{tabular}{|c|c|c|}
\hline & $M=16$ & $M=64$ \\
\hline$N t=4$ & $6+10$ & $8+14+20+22$ \\
$N r=2$ & $G_{v s-16 \mathrm{QAM}}=0.8198$ & $G_{v s-64 \mathrm{QAM}}=0.8355$ \\
\hline$N t=4$ & $6+10$ & $7+13+19+25$ \\
$N r=4$ & {$[0.5926,1.1787]$} & {$[0.3513,0.6557,0.9604,1.2652]$} \\
& $G_{v s-16 \mathrm{QAM}}=0.6312$ & $G_{v s-64 \mathrm{QAM}}=0.7389$ \\
$N t=4$ & $5+11$ & $7+13+19+25$ \\
$N r=8$ & {$[0.5302,1.1519]$} & {$[0.3513,0.6557,0.9604,1.2652]$} \\
& $G_{v s-16 \mathrm{QAM}}=0.4602$ & $G_{v s-64 \mathrm{QAM}}=0.6164$ \\
\hline
\end{tabular}

Wherein, $M$ accounts for the cardinality of multi-ring PSK constellation, $\mathrm{Nt}$ and $\mathrm{Nr}$ represent the number of transmit and receive antennas, respectively. The sequence $N_{1}+N_{2}+N_{3}+\ldots+N_{r}$ accounts for that multi-ring PSK contains $r$ rings and with the $i$ th $(i=1,2, \ldots, r)$ ring including $N_{i}$ constellation points. The vector $\left[R_{1}, R_{2}, \ldots, R_{r}\right]$ gives the radius of each ring with $R_{i}$ denoting the radius of the $i$ th $(i=1,2, \ldots, r)$ ring.

Furthermore, the performance gain of new designed multiring PSK over traditional PSK or QAM is given by the ratio of their $G_{N r}(M)$ values, which is denoted as $G_{v s-X X X X}$ with the subscript ${ }_{X x x x}$ representing traditional PSK or QAM. For instance, $G_{v s-16 Q A M}$ is the ratio of $G_{N r}(M)$ between new designed multi-ring PSK and traditional 16QAM. Obviously, the smaller the ratio, the better performance could be achieved. From table 1, it could be easily known that in most cases $G_{v s-X X X X}$ is less than one, i.e., the better performance of novel multi-ring PSK constellation could be achieved over traditional PSK or QAM.

\section{Simulation RESUlTS}

In this section, the SER (Symbol Error Rate) performance of different digital modulation schemes with $M=16$ is 
simulated over independent Rayleigh fading channels. The simulation results are shown in figure 2 . Wherein, MR-PSK denotes the novel designed multi-ring PSK constellation as shown in table 1, 16PSK and 16QAM represent traditional PSK and QAM constellations, respectively. From figure 2, we could easily see that the SER performance of novel designed multi-ring PSK is superior to traditional PSK or QAM.

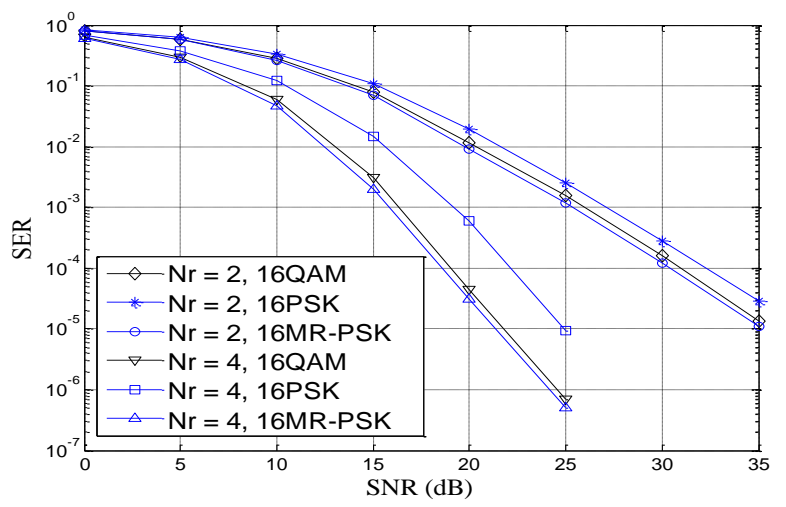

FIGURE II. SER PERFORMANCE OF MULTI-RING PSK CONSTELLATION.

\section{CONCLUSIONS}

In this paper, one new APM constellation design method for SM transmission is proposed. In order to facilitate the design process as well as without loss of generality, several design criteria are provided. The new designed APM constellation is called as multi-ring PSK constellation, just because it has multi-ring structure with each ring having PSK characteristics. Both analysis and simulation results have shown that in most cases the novel designed multi-ring PSK constellation could achieve better performance than traditional PSK or QAM in independent Rayleigh fading channels.

\section{ACKNOWLEDGEMENTS}

This research was partly supported by the National Natural Science Foundation of China (No. 61471269), the Doctoral Scientific Research Fund Project of Weifang University of China (No. 2014BS12) and Weifang Science and Technology Development Plan Project (No. 2014GX021).

\section{REFERENCES}

[1] Mesleh R., Haas H., Sinanovic S., Ahn C. W. \& Yun S., Spatial modulation. IEEE Transactions on Vehicular Technology, 57(4), pp. 2228-2241, Jul. 2008.

[2] Di Renzo M., Haas H. \& Grant P. M., Spatial modulation for multipleantenna wireless systems: A survey. IEEE Communications Magazine, 49(12), pp. 182-191, Dec. 2011.

[3] Di Renzo M., Haas H., Ghrayeb A., Sugiura S. \& Hanzo L., Spatial modulation for generalized MIMO: challenges, opportunities and implementation. Proceedings of the IEEE, 102(1), pp. 56-103, Jan. 2014.

[4] Yang P., Di Renzo M., Xiao Y. \& Li S., Design guidelines for spatial modulation. IEEE Communications Surveys \& Tutorials, accepted for publication.

[5] Di Renzo M. \& Haas H., Bit error probability of spatial modulation (SM-) MIMO over generalized fading channels. IEEE Transactions on Vehicular Technology, 61(3), pp. 1124-1144, Mar. 2012.

[6] Yang P., Xiao Y., Zhang B. \& Li S., Star-QAM signalling constellations for spatial modulation. IEEE Transactions on Vehicular Technology, 63(8), pp. 3741 - 3749, Oct. 2014.
[7] Maleki M., Bahrami H. R., Alizadeh A. \& Tran N. H., On the performance of spatial modulation: optimal constellation breakdown. IEEE Transactions on Communications, 62(1), pp. 144-157, Jan. 2014.

[8] Luna-Rivera M., Campos-Delgado D. U. \& Gonzalez-Perez M. G., Constellation design for spatial modulation. Procedia Technology, 7, pp. 71-78, 2013.

[9] Sugiura S., Xu C., Ng S. X. \& Hanzo L., Reduced complexity coherent versus non-coherent QAM-aided space-time shift keying. IEEE Transactions on Communications, 59(11), pp. 3090-3101, Nov. 2011. 